Casos Clínicos

Arch. Esp. Urol., 60, 2 (201-206), 2007

\section{ESTENOSIS URETERAL BILATERAL PROGRESIVA E IDIOPÁTICA.}

Enrique de Diego Rodríguez, Alberto Hernádez Castrillo, Miguel Ángel Rado Velázquez y José María Gómez Ortega?.

Servicio de Urología y Servicio de Anatomía Patológica'. Hospital Comarcal de Laredo. Cantabria. España.

Resumen.- OBJETIVO: Presentar un caso de estenosis ureteral bilateral de evolución progresiva sin constatar causa etiológica responsable del cuadro.

MÉTODOS: Se diagnostica y trata una paciente de 73 años de edad que desarrolla de forma progresiva una estenosis bilateral distal de ambos uréteres, sin objetivarse la causa responsable.

RESULTADO: Intervenida quirúrgicamente la paciente, el estudio anátomo-patológico puso de manifiesto una estrechez ureteral bilateral debido a una fibrosis e inflamación crónicas e inespecíficas.

CONCLUSIONES: La estenosis ureteral idiopática es un cuadro raro, más si cabe si su evolución es progresiva y con afectación bilateral.
Enrique de Diego Rodríguez

Miguel de Unamuno, 6 - 5으

39012 Santander. Cantabria. (España).

e.diego@telefonica.net

Trabajo recibido: 17 de julio 2006.
Palabras clave: Estenosis ureteral. Idiopática. Bilateral.

Summary.- OBJECTIVE: To report one case of progressive bilateral ureteral stenosis without demonstrable etiologic cause.

METHODS: We diagnosed and treated a 73-year-old female patient who developed progressive bilateral distal ureteral stenosis without a demonstrable cause.

RESULTS: Surgery was carried out and the pathologic study showed a bilateral ureteral stenosis secondary to fibrosis and chronic unspecific inflammation.

CONCLUSIONS: Idiopathic ureteral stenosis is a rare clinical picture, more if progressive and bilateral.

Keywords: Ureteral stenosis. Idiopathic. Bilateral.

\title{
INTRODUCCIÓN
}

Los diferentes procesos que pueden originar la formación de una estenosis ureteral están asociados generalmente con isquemia del uréter afecto, traumatismos en intervenciones previas, fibrosis periureteral o neoplasias (1).

La estrechez ureteral puede ser producida por procesos benignos como: litiasis, fibrosis (aneurisma aórtico, endometriosis), infecciones (tuberculosis, esquistosomiasis), lesiones iatrogénicas (cirugía abierta, instrumentación endoscópica) o neoformaciones benignas del uréter. Existen procesos malignos capaces de originar una estenosis ureteral: neoformaciones malignas de la pared ureteral, tumores metastáticos de cérvix, ovario, colonrecto, próstata etc.

Descartadas las posibles causas etiológicas que pueden originar la estrechez ureteral, ésta se clasifica como idiopática, debiendo ser evaluadas cuidadosamente para excluir un proceso maligno inadvertido (1).

Presentamos el caso clínico de una paciente que desarrolla un cuadro de estenosis ureteral bilateral distal progresiva sin causa etiológica demostrable.

\section{CASO CLÍNICO}

Paciente de 73 años, mujer, remitida a consulta de Urología por cuadro de dolor lumbar derecho y microhematuria de 1 mes de evolución. 
Como antecedentes personales destacan haber sido intervenida de histerectomía por miomas hace 26 años y de una hernia inguinal izquierda hace 15 años. Diagnosticada en 1998 de mieloma múltiple lg $G$ Lambda estadio IA (quiescente), siguiendo revisiones periódicas en servicio de hematología sin seguir tratamiento alguno. HTA e hiperglucemia en tratamiento dietético. Antecedente de fenómeno de Raynaud en seguimiento por Reumatología. No tratamientos médicos en la actualidad.
Encontrándose la paciente totalmente asintomática desarrolla un cuadro de dolor lumbar derecho sin clara irradiación ni otra clínica acompañante.

La exploración física resulta anodina con una puñopercusión renal derecha levemente positiva.

El elemental y sedimento de orina mostraba la presencia de 60-80 hematíes por campo, el urocultivo resultó
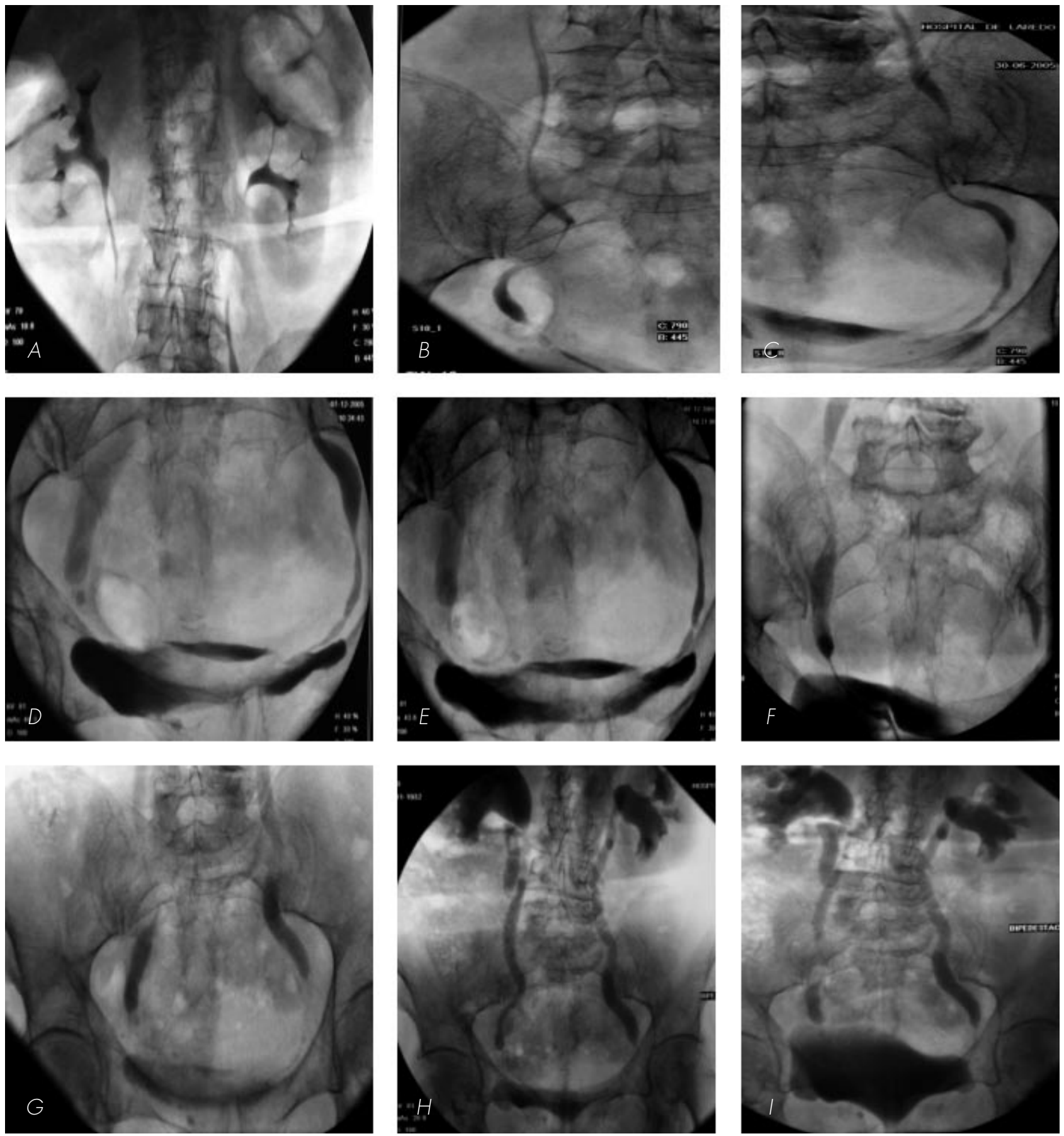

FIGURA 1. (A, B, y C): urografías iniciales.

$(D, E$ y $F)$ : estenosis ureteral distal derecha.

( $G, H$, I): estenosis ureteral distal bilateral. 
negativo y las citologías urinarias también resultaron negativas para células malignas.

El estudio ecográfico evidenció unos riñones y una vejiga totalmente normales y la urografía intravenosa puso de manifiesto unos riñones de tamaño, morfología y función normal, con una vía excretora permeable. (Figura $1 \mathrm{~A}, \mathrm{~B}$ y $\mathrm{C}$.)

A las 3 semanas la paciente acude de nuevo por exacerbación del dolor lumbar derecho y emisión de coágulos finos y alargados. Se practicó nuevo estudio citológico de orina que también resultó negativo y nueva urografía donde se evidenció una ureterohidronefrosis grado II secundaria a estenosis de uréter distal derecho (Figura $1 \mathrm{D}$ y E). Se realizó pielografía retrógrada derecha que confirmó la presencia de una estenosis distal de 2-3 cm. (Figua 1 F). Se tomó muestras de lavado de ese uréter para estudio citológico que resultaron negativas para malignidad.

Se pautó tratamiento antiinflamatorio, con una discreta mejoría clínica que vuelve a empeorar a los 2 meses, presentando la paciente en ese momento dolor lumbar bilateral, irradiado a flancos persistiendo la eliminación de coágulos filiformes. Se practicó nuevo estudio urográfico que esta vez demostró la presencia de una ureterohidronefrosis bilateral grado III, secundaria a estenosis distal de ambos uréteres (Figura 1 G, H, I). Se realizó T.A.C. abdómino-pélvico que descartó la existencia de procesos extrínsecos que originasen la estenosis, sólo evidenciando una luz ureteral puntiforme ocupada en ambos uréteres.

Se practicó cistoscopia cuidadosa que resultó normal y se tomaron muestras de lavado de ambos uréteres,
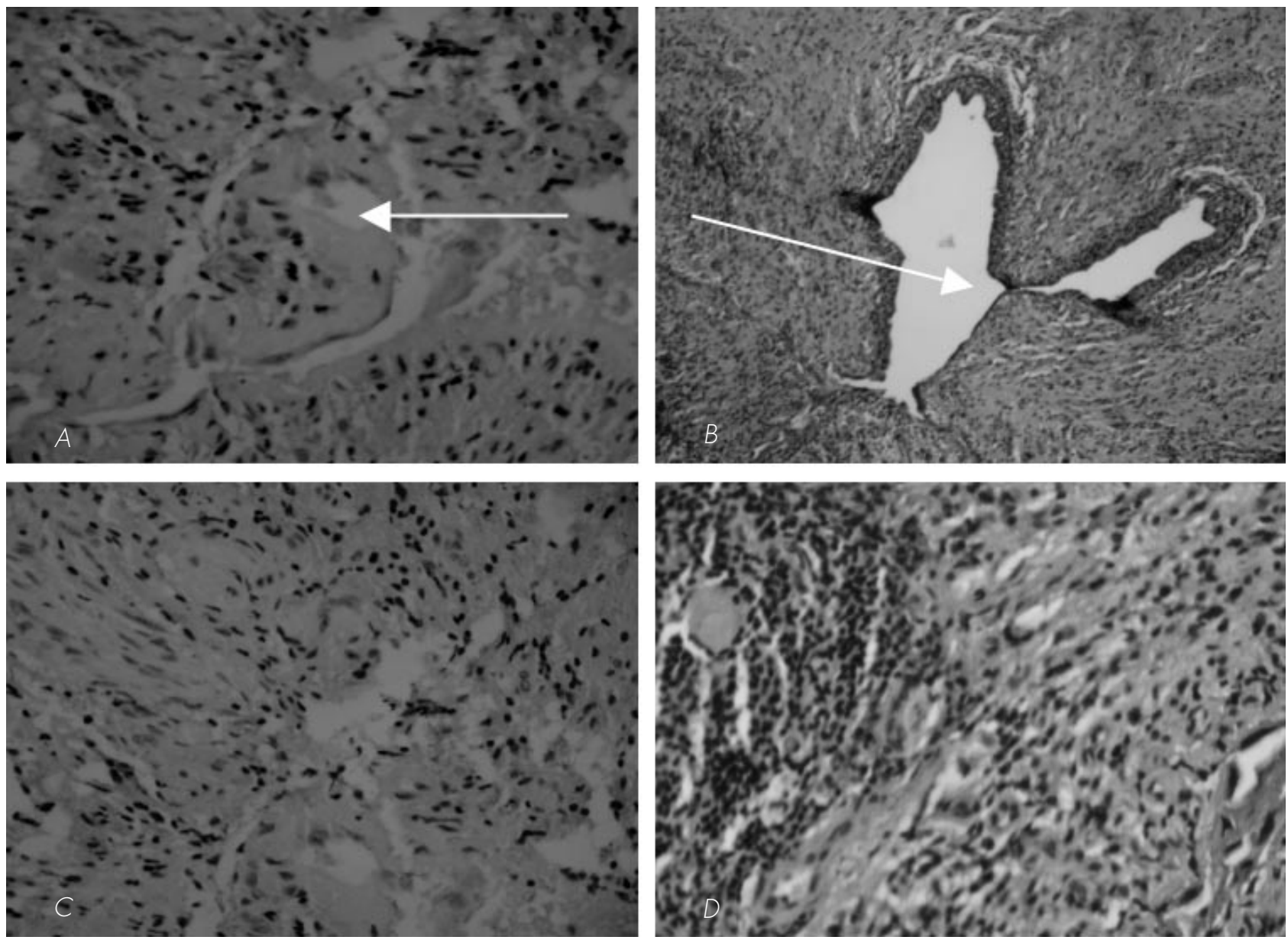

FIGURA 2. (A y B): uréteres derecho e izquierdo.

H.E. 200 y $40 x$.

(C y D): intenso componente inflamatorio.

H. E. $200 x$. 
con una cateterización bastante dificultosa, resultando el estudio citológico negativo.

Ante la evolución y bilateralidad del cuadro se decide intervención quirúrgica abierta, encontrándose unos uréteres normales macroscópicamente, sin encontrarse englobados en procesos fibrosos o adherenciales, remitiendo biopsia intraoperatoria del segmento estenótico que fue informada como oclusión de la luz ureteral por proceso inflamatorio agudo-crónico y fibrosis. Se realizó ureteroneocistotomía bilateral tipo Politano-Leadbetter.

El estudio anátomo-patológico definitivo confirmó la presencia de inflamación crónica, edema y fibrosis de la luz de ambos uréteres afectando sobre todo a la submucosa, sin evidenciarse granulomas, vasculitis o depósito de amiloide. (Figura 2)

A los 3 meses de seguimiento la paciente se encontraba asintomática y el estudio urográfico puso de manifiesto unos riñones normales, con unos uréteres permeables hasta su entrada en vejiga (Figura 3); a los 6 meses la enferma continua sin síntomas.

\section{DISCUSIÓN}

La estenosis bilateral de uréter es un proceso raro y más aún en ausencia de un factor etiológico que lo desencadene. (2)

En niños se ha descrito la estenosis ureteral congénita (3), que habitualmente suele ser unilateral. En el adulto, la fibrosis retroperitoneal, los aneurismas aórticos, quimioterapia $y / o$ radioterapia previa, lesiones iatrogénicas en cirugía abierta- endoscópica $\circ$ procesos neoplásicos locales o metastáticos, son posibles causas de estrecheces ureterales bilaterales. (2)

En la literatura se recogen causas más raras de estenosis bilateral de uréteres: candidiasis (4), bilharziasis (5), post-instilación de Mitomicina C (6), amiloidosis (7), poliarteritis nodosa (8) o ureteritis eosinofílica (9).

Actualmente el tratamiento de estas estenosis se puede realizar con técnicas mínimamente invasivas (10) cuando el caso lo permite, con resultados similares a la cirugía abierta.

En la revisión bibliográfica realizada no hemos encontrado ninguna referencia sobre estenosis ureteral bilateral de causa desconocida y de curso progresivo, sólo Bhatta (2) describe 2 casos de estenosis ureteral idiopática pero unilateral.

En nuestro caso, destacamos la peculiar evolución clínica de la paciente, no considerando que el antecedente de una cirugía previa desarrollada hace 26 años constituyese la causa desencadenante del cuadro. Descartadas las causas habituales, pensamos en algún proceso de naturaleza autoinmune, dados los antecedentes de la paciente (mieloma quiescente, fenómeno de Raynaud), pero el estudio histológico de las piezas quirúrgicas remitidas descartó la presencia de vasculitis, granulomas o depósito de amiloide, mostrando un intenso componente inflamatorio, fundamentalmente a nivel de la submucosa que ocluía la luz ureteral, no existiendo afectación extrínseca de la pared ureteral como se produciría en caso de una cirugía previa reciente.

Por último señalar que optamos por la cirugía abierta ante la falta de un diagnóstico etiológico, bilateralidad del cuadro y grado y longitud de las estenosis.
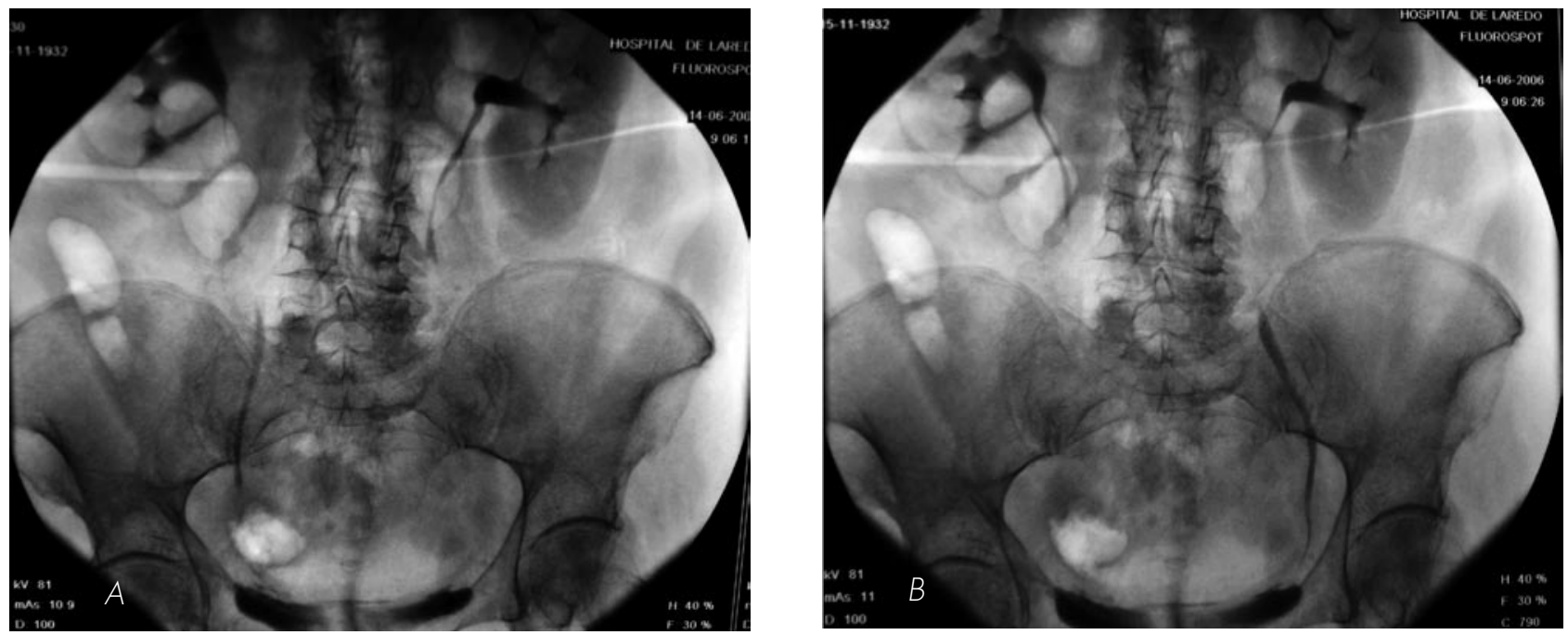

FIGURA 3. U.I.V. a los 3 meses. Riñones normales. Ausencia de estenosis ureteral. 


\section{BIBLIOGRAFÍA y LECTURAS RECOMENDADAS ( ${ }^{*}$ lectura de interés $y^{* *}$ lectura fundamental)}

**1. STEVAN, B.; STREEM,; JENNY, J. y cols.: “Manejo de la obstrucción de las vías urinarias superiores". En: Patrick C. Walsh; Alan, B.; Retik, E. y cols. (eds). Campbell Urología. 8a edición. 503-555. Panamericana. Buenos Aires. 2004.

**2. BHATTA DHAR, N.; ANGERMEIER, K.W.; STREEM, S.B. y cols.: "Idiopathic ureteral strictures without evidence of malignancy". Urology, 64: 377, 1994.

3. HWANG, A.H.; McALEER, I.M.; SHAPIRO, E. y cols.: "Congenital mid ureteral strictures". J. Urol. 174: 1999, 2005.

4. LYE, W.C.; LEE, E.J.; TUNG, K.H. y cols.: "Bilateral ureteric strictures secondary to candidiasis". Br. J. Urol. 67: 551, 1991.

5. AL-SHUKRI, S.; ALWAN, M.H.: "Bilharzial strictures of the lower third of the ureter: a critical review of 560 strictures". Br. J. Urol. 55: 477, 1983.

6. WASS, A.R.; LAWSON, T.R.; URWIN, G.H.: "Bilateral ureteric strictures after intravesical mitomicyn C". Br. J. Urol. 72: 661, 1993.

7. FUSHIMI, T.; TAKEI, Y.; TOUMA, T. y cols.: "Bilateral localized amyloidosis of the ureters: clinicopathology and therapeutic approaches in two cases". Amiloid. 11: 260, 2004.

8. HEFTY, TR.; BONAFEDE, P.; STENZEL, P.: "Bilateral ureteral stricture from polyarteritis nodosa". J.Urol. 141: 600, 1989.

9. SINGH, J.C.; KARNIK, SV.; GOPALAKRISHANAN, G.: "Eosinophilic ureteritis presenting as bilateral upper ureteric strictures". Scand. J. Urol. Nephrol. 38: 260, 2004.

*10. HAFEZ, K.S.; WOLF, J.S.: "Update on minimally invasive management of ureteral strictures". J. Endourol. 17: 453, 2003.

\section{Casos Clínicos}

Arch. Esp. Urol., 60, 2 (205-207), 2007

\section{TUMOR TESTICULAR BILATERAL SINCRÓNICO CON FOCOS DE NEOPLASIA INTRATUBULAR DE CÉLULAS GERMINALES: PRESENTACIÓN DE UN CASO.}

Jorge Planelles Gómez, Aleixandre Vergés Prósper, Ignacio Rubio Tortosa, Manel Beamud Cortés, Teresa Pastor Navarro, José Ramón Beltrán Armada y

Carlos San Juan de Laorden

Servicio de Urología. Hospital Universitario Doctor Peset. Valencia. España.

Resumen.- OBJETIVO: Presentar un caso de tumor testicular bilateral sincrónico con focos de Neoplasia Intratubular de Células Germinales (NICG) en ambos testículos.

MÉTODOS: Describimos el caso de un varón de 29 años que presenta tumoración indolora de un mes de evolución en teste izquierdo. Bajo la sospecha de tumor testicular se le realiza una ecografía escrotal, una determinación de marcadores tumorales y un estudio de extensión tumoral.

RESULTADOS: En la ecografía se puede ver un teste izquierdo hipotrófico con lesión sospechosa de neoplasia $e$, incidentalmente, un testículo derecho con una lesión hipoecoica y aumento de flujo doppler. Se le practicó una orquiectomía bilateral con el diagnóstico de anatomía patológica de seminoma clásico con focos de Neoplasia Intratubular de Células Germinales (NICG) en ambos testículos y se completó el tratamiento con 2 ciclos de quimioterapia.

| Jorge Planelles Gómez

Servicio de Urología

Hospital Universitario Doctor Peset

Gaspar Aguilar 90

46017 Valencia. (España).

jorge_planelles@yahoo.es

Trabajo recibido: 18 de julio 2006. 